03

\title{
Анализ развития возмущений в гиперзвуковом пограничном слое за волнистой поверхностью
}

\author{
(ㄷ Д.А. Бунтин ${ }^{1,2}$, А.А. Маслов ${ }^{1,2}$ \\ ${ }^{1}$ Институт теоретической и прикладной механики им. С.А. Христиановича \\ СО РАН, Новосибирск \\ ${ }^{2}$ Новосибирский государственный университет \\ E-mail: bountin@itam.nsc.ru
}

Поступило в Редакцию 9 марта 2017 г.

Экспериментально исследована устойчивость сверхзвукового пристенного течения над мелко гофрированной пластиной в набегающем потоке аэродинамической трубы с числом Маха 6. Получены коэффициенты роста возмущений, которые показывают, что волнистая стенка стабилизирует течение в пограничном слое относительно возмущений второй моды, но дестабилизирует возмущения первой моды. Проведен биспектральный анализ возмущений. Получено, что с уменьшением амплитуд пульсаций второй моды нелинейные процессы в пограничном слое за волнистой поверхностью становятся сильно подавленными: гармонический резонанс второй моды отсутствует, а нелинейные процессы в низкочастотной области спектра имеют на порядок более низкую амплитуду взаимодействия и существенно более ограниченный частотный диапазон. Благодаря стабилизирующему влиянию волнистой поверхности на возмущения второй моды начало ламинарно-турбулентного перехода в целом задерживается.

DOI: 10.21883/PJTF.2017.13.44813.16733

Ламинарно-турбулентный переход (ЛТП) приводит к существенному росту аэродинамического сопротивления и нагрева поверхности, а также к уменьшению эффективности двигательной установки гиперзвуковых аппаратов. Возможность удлинения ламинарного участка важна при разработке и оптимизации перспективных аэрокосмических самолетов. При гиперзвуковых скоростях ЛТП вызывается возмущениями низкочастотной первой (вихревой) и высокочастотной второй (акустической) модами возмущений. Их частотные диапазоны различаются 
примерно в два раза. Подавление неустойчивых возмущений этих мод может привести к задержке ЛТП и удлинению ламинарного участка. В данной работе изучена возможность подавления наиболее опасной для турбулизации течения второй моды с помощью гофрированной поверхности.

Известно, что увеличение числа Маха оказывает стабилизирующий эффект на течение в свободных слоях смешения и спутных следах (например, [1]). Естественно предположить, что относительно длинный свободный слой смешения, сформированный около обтекаемой поверхности, может уменьшить темпы роста и подавить возмущения второй моды, имеющие короткую длину волны (порядка двух толщин пограничного слоя). Эта гипотеза была подтверждена численно в работе [2]. Экспериментальные спектры возмущений качественно совпали с расчетами [3]. Однако, чтобы в полной мере говорить о стабилизирующем влиянии волнистой поверхности, необходимо понимать, как такая поверхность влияет на нелинейные взаимодействия в пограничном слое (которые в конечном итоге приводят к турбулентности) и как ведут себя коэффициенты роста возмущений. В настоящем экспериментальном исследовании были получены коэффициенты роста возмущений и проведен биспектральный анализ нелинейных процессов в пограничном слое на сплошной и за волнистой стенками.

Эксперименты были проведены в импульсной аэродинамической трубе „Транзит-М“ ИТПМ СО РАН при числе Маха набегающего потока 6. Единичные числа Рейнольдса составляли $\operatorname{Re}_{1 \infty}=9.3,11.3,12.4$ и $14 \cdot 10^{6} 1 / \mathrm{m}$; при этом давление и температура торможения менялись в диапазоне $P_{0}=0.7-1.1 \mathrm{MPa}, T_{0}=360-375 \mathrm{~K}$. Экспериментальной моделью была плоская пластина из нержавеющей стали с острой передней кромкой. Длина пластины $350 \mathrm{~mm}$, ширина $200 \mathrm{~mm}$ и толщина $15 \mathrm{~mm}$ (рис. 1). Область гофрированной поверхности состоит из 9 углублений в виде дуг окружностей. Длина по размаху гофрированной области равна половине размаха пластины. Пульсации в пограничном слое измерялись быстродействующими датчиками ALTP, имеющими частотный диапазон до $1 \mathrm{MHz}$ [4]. Для установки датчиков модель имеет несколько технологических отверстий, как это показано на рис. 1. Во время каждого пуска измерения на плоской и за гофрированной областями проводились одновременно.

Для вычисления коэффициентов роста возмущений вниз по потоку для случая двух датчиков, расположенных один за другим, использо-

Письма в ЖТФ, 2017, том 43, вып. 13 

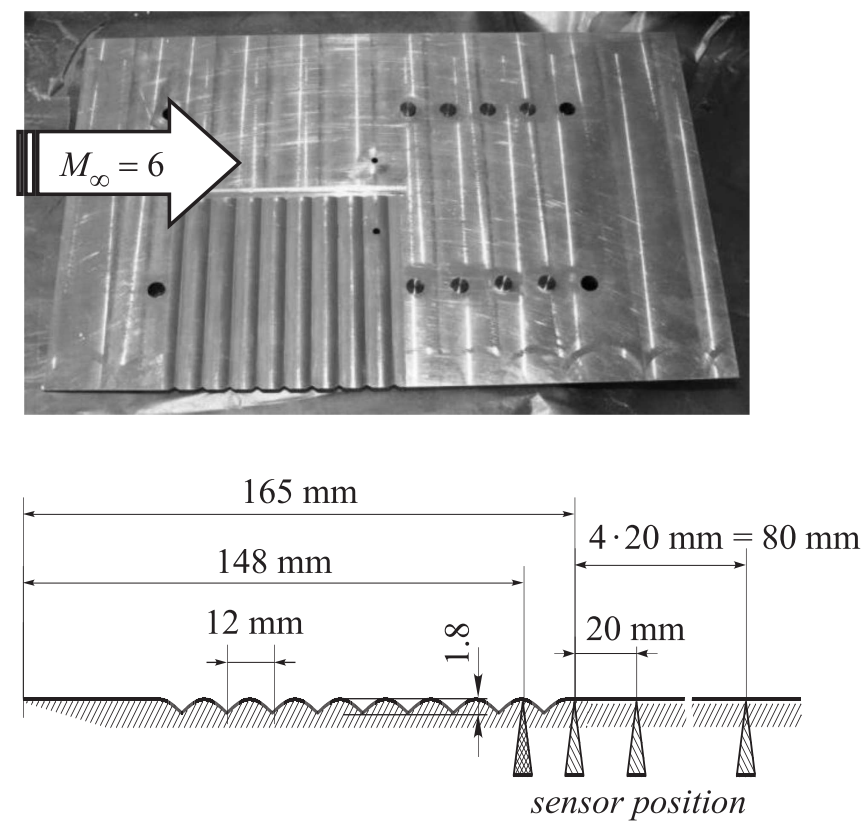

Рис. 1. Фотография и схема модели.

валась формула [5]: $-\alpha_{i}=\left(\frac{\ln \left(A_{1} / A_{2}\right)}{2\left(R_{1}-R_{2}\right)}\right)$, где отрицательные значения $\alpha_{i}$ обозначают рост возмущений; $R=\sqrt{\mathrm{Re}_{e x}} ; \mathrm{Re}_{e x}$ - число Рейнольдса, рассчитанное по значениям на границе пограничного слоя и расстоянию от носика модели до датчика; $A_{1}$ и $A_{2}-$ амплитуды волн фиксированной частоты, измеренные на 1 -м и 2-м датчиках, $R_{1}$ и $R_{2}-$ параметр $R$, посчитанный для координат 1-го и 2-го датчиков.

На рис. 2 показаны степени роста возмущений для режимов течения с единичными числами Рейнольдса $\mathrm{Re}_{1}=9.3$ и $11.3 \cdot 10^{6} 1 / \mathrm{m}$. Степени роста возмущений получены по измерениям двух соседних датчиков, находящихся в положениях $x=165 \mathrm{~mm}(R=1239)$ и $x=185 \mathrm{~mm}$ $(R=1312)$. Графики коэффициентов роста приведены в зависимости от размерной частоты (нижняя шкала) и от безразмерной частоты $f x / U_{e}\left(\operatorname{Re}_{e x}\right)^{1 / 2}$ (верхняя шкала), где $f$ - частота; $x$ - расстояние от

5* Письма в ЖТФ, 2017, том 43, вып. 13 

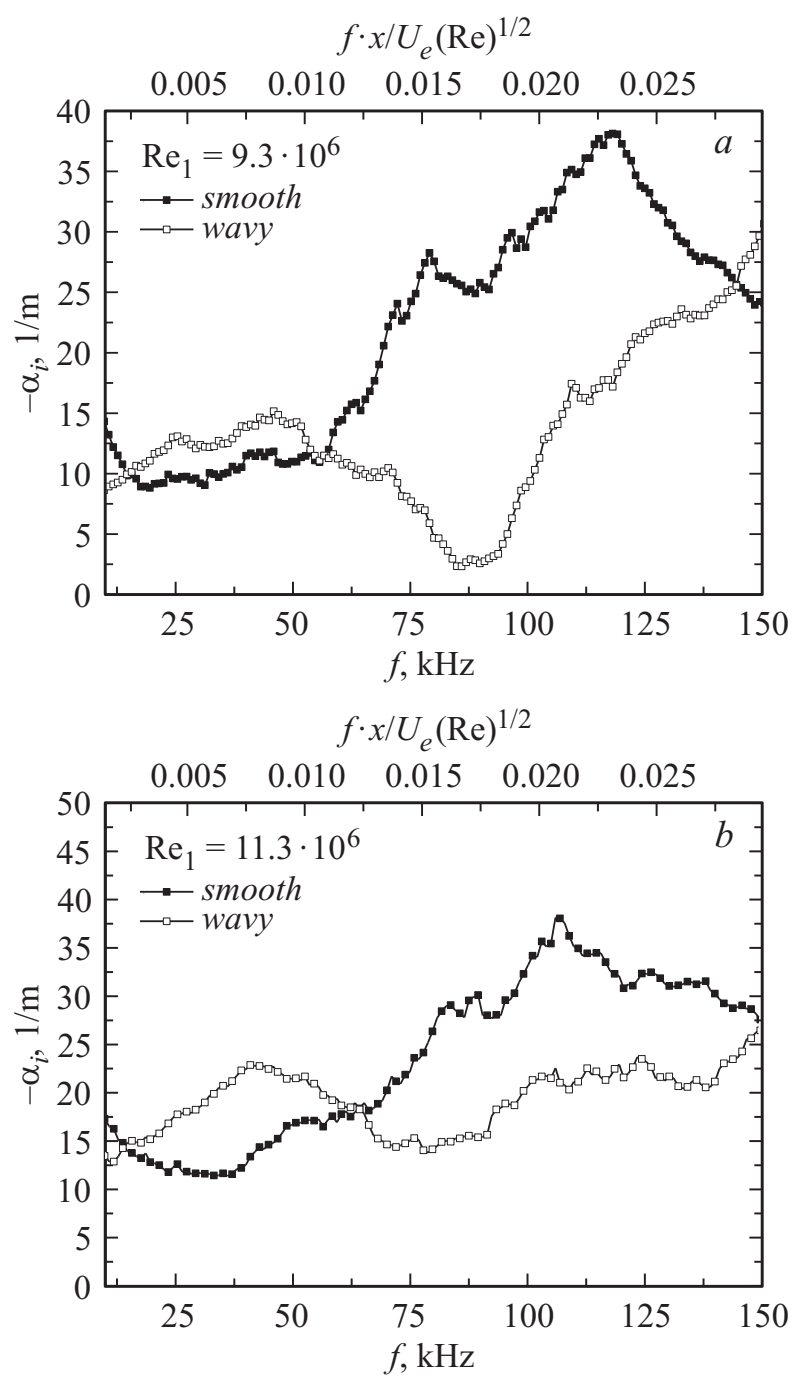

Рис. 2. Степени роста: $a-\operatorname{Re}_{1}=9.3 \cdot 10^{6} 1 / \mathrm{m} ; b-\operatorname{Re}_{1}=11.3 \cdot 10^{6} 1 / \mathrm{m}$. 
передней кромки пластины до первого датчика; $U_{e}-$ скорость потока на границе пограничного слоя.

На верхних графиках рис. 3 над диаграммами бикогерентности показаны Фурье-спектры исследовавшихся сигналов. При $f=150 \mathrm{kHz}$ в спектрах наблюдаются пики. Длина волны возмущения для них примерно равна $0.4 \mathrm{~mm}$ при толщине пограничного слоя около $0.18 \mathrm{~mm}$, т. е. их отношение равно примерно двум, что характерно для второй моды возмущений. Безразмерный частотный параметр также соответствует второй моде.

Из рис. 2 видно, что значение коэффициентов роста второй моды за волнистой поверхностью в диапазоне $75-150 \mathrm{kHz}$ лежат существенно ниже значений, полученных на гладкой поверхности. Таким образом, рост возмущений второй моды за волнистой поверхностью замедлен по отношению к росту на гладкой поверхности. Наименьшие значения коэффициентов роста соответствуют возмущениям с частотами $75-80 \mathrm{kHz}$. Эти возмущения являются результатом влияния волнистости на среднее течение. При $\operatorname{Re}_{1}=9.3 \cdot 10^{6} 1 / \mathrm{m}$ коэффициенты роста для таких возмущений близки к 0 , т. е. эти возмущения практически не растут вниз по течению (рис. 2,a).

Следует отметить, что возмущения с частотами в диапазоне $25-50 \mathrm{kHz}$ за волнистой поверхностью растут быстрее, чем в случае сплошной поверхности. Однако спектры пульсаций показывают (рис. 3, верхние графики), что амплитуды возмущений на этих частотах за волнистой поверхностью не превышают значений амплитуд над гладкой поверхностью, т.е. они не могут вызвать различия в поведении ЛТП. Для $\operatorname{Re}_{1}=11.3 \cdot 10^{6} 1 / \mathrm{m}$ максимальная амплитуда в спектрах для гладкой поверхности (рис. $3, b)$ соответствует частоте $f=150 \mathrm{kHz}$, а максимальные коэффициенты роста соответствуют частоте $f=110 \mathrm{kHz}$ (рис. $2, b$ ), т.е. низкочастотная часть спектра второй моды растет быстрее, смещая максимум в спектрах в низкочастотную область. За волнистой поверхностью в диапазоне $100-125 \mathrm{kHz}$ коэффициенты роста второй моды практически постоянны и форма спектра не изменяется.

Для анализа нелинейных взаимодействий сигналы датчиков обрабатывались с помощью биспектрального преобразования. Спектры полученных коэффициентов $\mathrm{bic}^{2}$ количественно отражают степень нелинейных взаимодействий квадратичного типа трех волн с частотами $f_{1}, f_{2}, f_{3}=f_{1}+f_{2}[6]$.

Письма в ЖТФ, 2017, том 43, вып. 13 

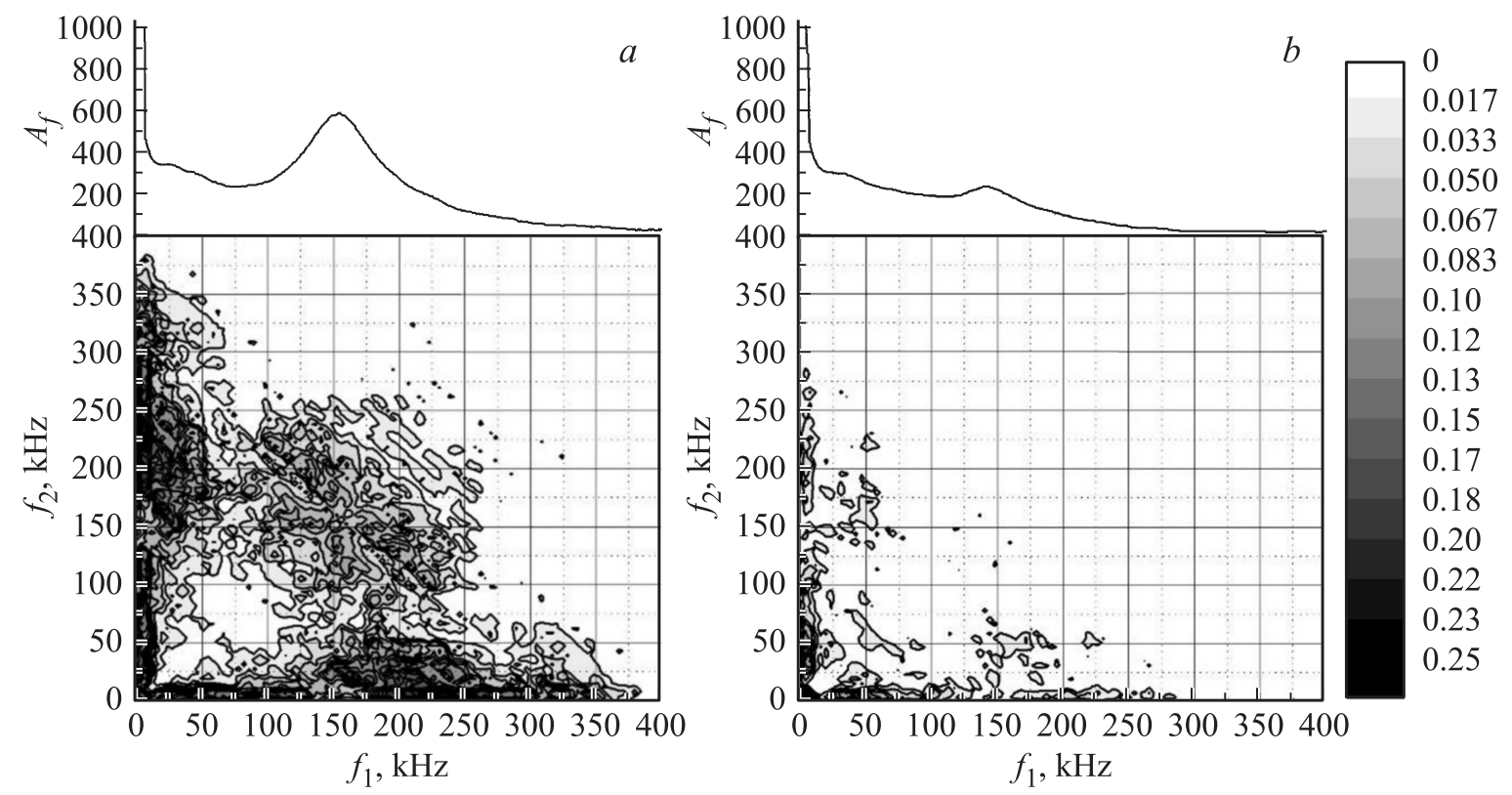

Pиc. 3. Диаграммы бикогерентности, полученные по сигналам датчиков ALTP: $a-$ на гладкой поверхности; $b-$ за волнистой поверхностью. $\mathrm{Re}_{1}=11.3 \cdot 10^{6} \mathrm{1} / \mathrm{m}$. Сверху приведены фурье-амплитуды пульсаций. 
Анализ показал, что сразу за координатой волнистой поверхности (в положении датчика $x=165 \mathrm{~mm}, R=1239$ ) на гладкой поверхности наблюдается область слабого нелинейного взаимодействия чуть выше уровня шумов $\left(b_{i} c^{2} \approx 0.04\right)$. За волнистой поверхностью такое взаимодействие отсутствует.

Однако уже в следующем положении датчиков $(x=185 \mathrm{~mm}$, $R=1312)$ на гладкой поверхности на диаграмме наблюдается очень широкое взаимодействие волн разных частот (рис. $3, a$ ): взаимодействие второй моды $f_{1}=125-350 \mathrm{kHz}$ с низкочастотными возмущениями $f_{2}=1-60 \mathrm{kHz}$; взаимодействие волн с низкими частотами $\left(f_{1}=1-125\right.$ и $\left.f_{1}=1-25 \mathrm{kHz}\right)$.

Интенсивность нелинейного взаимодействия в данном положении датчика возрастает до значения $b i c^{2} \approx 0.3$. Кроме этого, на диаграмме наблюдается обширная область взаимодействия на частотах $f_{1,2}=100-200 \mathrm{kHz}$, соответствующих частотам возмущений второй моды (рис. $3, a)$. Взаимодействие $\left(f_{1,2}=f_{\mathrm{II}}, f_{3}=f_{1}+f_{2}=2 f_{\text {II }}\right)$ является гармоническим резонансом второй моды $\left(b i c^{2} \approx 0.1\right)$, т. е. приводит к росту гармоники волн второй моды. Данный тип взаимодействия является типичным в гиперзвуковом пограничном слое [7]. Бурное проявление нелинейных процессов говорит о начале турбулизации потока. Интенсивность гармонического резонанса приблизительно в 2 раза ниже интенсивности взаимодействия второй моды с низкочастотными возмущениями. Датчик, находящийся за волнистой поверхностью в этом же сечении $(x=185 \mathrm{~mm}, R=1312)$, показывает, что гармонический резонанс второй моды отсутствует, а нелинейные процессы в низкочастотной области спектра существенно ниже как по амплитуде, так по частотному диапазону (рис. $3, b$ ). Таким образом, за волнистой поверхностью турбулизация потока еще не наступила.

Таким образом, в работе впервые выполнен анализ развития возмущений в гиперзвуковом пограничном слое за волнистой стенкой и проведено сравнение с эволюцией возмущений над гладкой поверхностью плоской пластины. Сравнение степеней роста пульсаций показало, что волны второй моды за волнистой поверхностью растут существенно медленнее, чем в случае сплошной поверхности. Следует отметить, что низкочастотные возмущения $(15-60 \mathrm{kHz})$ за волнистой поверхностью растут быстрее, чем в случае сплошной поверхности, но их амплитуда сравнима с возмущениями на плоской поверхности, и они не могут изменить сценарий перехода.

Письма в ЖТФ, 2017, том 43, вып. 13 
Биспектральный анализ данных показывает, что интенсивность нелинейных процессов в пограничном слое за волнистой поверхностью на порядок ниже, чем для гладкой поверхности: сильный гармонический резонанс, хорошо видимый в пограничном слое на сплошной поверхности, полностью отсутствует; интенсивные нелинейные процессы в низкочастотной области спектра имеют на порядок более низкую амплитуду взаимодействия и существенно более ограниченный частотный диапазон. Таким образом, нелинейные процессы на волнистой поверхности отстают в своем развитии от процессов на сплошной поверхности.

Благодаря стабилизирующему влиянию волнистой поверхности на возмущения второй моды начало ламинарно-турбулентного перехода в целом задерживается.

Работы выполнена при поддержке РФФИ, грант 16-08-00782.

\section{Список литературы}

[1] Lysenko V.I. // J. Fluid Mech. 1999. V. 392. P. 1-6.

[2] Egorov I.V., Novikov A.V., Fedorov A.V. // 48th AIAA Aerospace Sciences Meeting. 2010.

[3] Bountin D., Chimitov T., Maslov A. et al. // AIAA Journal. 2013. V. 51. P. 12031210.

[4] Бобамев С.В, Менде Н.П., Попов П.А. и др. // Письма в ЖТФ. 2009. Т. 35. B. 5. C. $36-42$.

[5] Roediger T., Knauss H., Estorf M. et al. // J. Spacecraft Rockets. 2009. V. 46. N 2. P. $266-273$.

[6] Бунтин Д.А., Маслов А.А., Миронов С.Г. и др. // ПМТФ. 2012. Т. 53. № 1. C. 3-11.

[7] Bountin D., Shiplyuk A., Maslov A. // J. Fluid Mech. 2008. V. 611. P. 427-442. 